\title{
Scattering Fields Control by Metamaterial Device Based on Ultra-Broadband Polarization Converters
}

\author{
Si-Jia $L I^{1}$, Li-Ming $X U^{2}$, Xiang-Yu $C A O^{1}$, Jiang-Feng $H A N^{1}$, \\ Zhao ZHANG ${ }^{l}$, Xiao LIU ${ }^{l}$, Kui-Sheng FENG ${ }^{l}$, Chen ZHANG ${ }^{l}$ \\ ${ }^{1}$ Information and Navigation College, Air Force Engineering University, No.1 FengHao Road, Xi'an 710077, China \\ ${ }^{2}$ Science and Technology on Electronic Information Control Laboratory, Chendu 610036, China
}

1sj051@126.com,10575229@qq.com,gjgj9694@163.com,hanjimk1@163.com,bjzhangzhao323@126.com, 382500638@qq.com, fks98112@163.com,xue3201ong@sina.cn

Manuscript received June 11, 2016

\begin{abstract}
We proposed a novel ultra-broadband metamaterial screen with controlling the electromagnetic scattering fields based on the three layers wideband polarization converter (TLW-PC). The unit cell of TLW-PC was composed of a three layers substrate loaded with double metallic split-rings structure and a metal ground plane. We observed that the polarization converter primarily performed ultra-broadband cross polarization conversion from $5.71 \mathrm{GHz}$ to $14.91 \mathrm{GHz}$. Furthermore, a metamaterial screen, which contributed to the low scattering characteristics, had been exploited with the orthogonal array based on TLW-PC. The near scattering electronic fields are controlled due to the change of phase and amplitude for incident wave. The metamaterial screen significantly exhibited low scattering characteristics from $5.81 \mathrm{GHz}$ to 15.06 GHz. To demonstrate design, a metamaterial device easily implemented by the common printed circuit board method has been fabricated and measured. Experimental results agreed well with the simulated results.
\end{abstract}

\section{Keywords}

Ultra-broadband, radar cross section reduction, metamaterial, polarization converter

\section{Introduction}

In recent years, great efforts have been dedicated to the metamaterial focusing in microwave, terahertz and optical frequencies, which inspired many applications such as acoustic cloaks [1-3], gradient index lenses, hyperlenses, perfect absorbers, polarization rotators, and many other devices [4-9]. As focusing points, near and far scattering electromagnetic fields were controlled by metamaterial. Especially, the broadband and low far-field scattering such as radar cross section (RCS) have been adequately paid attention to due to the stealth of platform. To obtain low RCS, perfect metamaterial absorber (PMA) with near- unity absorptivity and ultrathin structure was firstly proposed by Landy et al. [10], which had become an important research aspect of the metamaterials. Later, researchers made efforts on the different PMA structures to achieve broadband absorption with insensitive polarization [11-15] Another way of achieving low RCS using a planar configuration was proposed by M. Paquay [16]. The planar structure, based on a combination of artificial magnetic conductors (AMC) and perfect conductors in a chessboard like configuration, has shown the possibility of narrow band RCS reduction due to the destructive interference of phase. Then different combinations of several AMCs structures were proposed for broadband RCS reduction [17], [18].

Importantly, metamaterials were used to realize the circular polarizers, polarization rotators or metasurface with asymmetric transmission to manipulate polarization of the electromagnetic (EM) waves [19-21]. The plasmon resonances and plasmon hybridizations were observed in metamaterials and were applied for manipulation of polarization recently [22]. The plasmon hybridization could occur within not only a single structure but also a complex structure if the structure supported the multiple plasmon resonances and was observed in metamaterial structures at microwave wavelengths [23], [24]. More recently, the metasurfaces with different AMCs or converters have been paid attention to because of the outstanding performance for controlling the scattering fields [25-27]. On this basis, we proposed a three layers wideband polarization converter (TLW-PC) using metamaterials in this contribution. The three layers cross polarization converter manipulated the polarization of the incident waves from $5.71 \mathrm{GHz}$ to 14.91 GHz. Moreover, a metamaterial screen device was designed based on the proposed polarization converter. The device performed ultra-broadband low scattering because of destructive interference of the phase and the amplitude. The experimental data and the simulated results indicated that the metamaterial screen device performed attractive RCS reduction comparing to the designed converter. This metamaterial device provided an effective method for ultrabroadband low scattering especially RCS reduction. 


\section{Design and Analysis of TLW-PC}

As we all know, the polarization dependent effects can be provided by the anisotropic structures. The polarization states can be manipulated by the interference of the polarization dependent reflections for the proposed structure. The ring cavity is systemically proved to support different plasmon hybridizations [22-24]. On this basis, the polarization converter is designed and measured. The unit cell of the polarization converter is composed of a threelayers substrates with double metallic split-rings (DMSRs) structure and copper ground plane as shown in Fig. 1. The substrates are all Arlon $\operatorname{AD} 430\left(\varepsilon_{\mathrm{r}}=4.3\right.$ and $\left.\tan \delta=0.003\right)$, and their thicknesses are $1.5 \mathrm{~mm}, 1.5 \mathrm{~mm}$ and $1 \mathrm{~mm}$, respectively. The conductivity of copper is $5.8 \times 10^{7} \mathrm{~S} / \mathrm{m}$ and the thickness is $36 \mu \mathrm{m}$. The geometrical parameters of the DMSRs are shown as follows: the periodicity $l=10 \mathrm{~mm}$, the outside length of DMSR-1 $r_{1}=3.42 \mathrm{~mm}$, the outside length of DMSR-2 $r_{3}=4.6 \mathrm{~mm}$, the inside length of DMSR-1 $r_{2}=2.92 \mathrm{~mm}$ and the inside length of DMSR-2 $r_{4}=4.1 \mathrm{~mm}$. The splits widths of the DMSRs structures are all $w_{1}=w_{2}=0.5 \mathrm{~mm}$.

We propose the polarization converter operating in the refection mode at gigahertz frequency. Based on the concept of metamaterials or metasurface, the DMSRs structure and copper ground in conjunction rotate the linear polarization by $90 \mathrm{deg}$ within an extended operation bandwidth. The underlying physical mechanism of the polarization converter and the screen with low RCS can be investigated through decomposed electric field components that interact with the DMSRs structure. For verifying polarization converter, numerical simulations are performed using the software HFSS. In order to demonstrate the polarization conversion efficiency, the polarization conversion ratio $(P C R)$ is introduced and defined as

$$
P C R=\frac{R_{\mathrm{xy}}}{R_{\mathrm{xy}}+R_{\mathrm{xx}}}=\frac{\left|S_{\text {cross }-11}\right|^{2}}{\left|S_{\text {cross-11 }}\right|^{2}+\left|S_{\mathrm{co}-11}\right|^{2}}
$$

where $R_{\mathrm{xy}}$ and $R_{\mathrm{xx}}$ are reflectance of the cross-polarized and co-polarized waves. $S_{\text {cross-11 }}$ and $S_{\text {co-11 }}$ are the reflected coef-

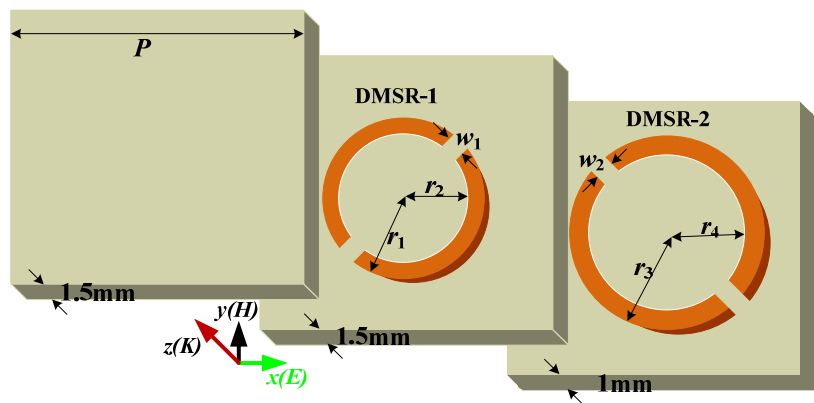

Fig. 1. Geometry of unit cell for the polarization conversion The cell denoted as element " 0 " which is composed of three substrate layers, double metallic split-rings (DMSRs) structure and a metal ground plane. The underside of the first layer, middle layer and bottom layer is respectively DMSR-1, DMSR-2 and copper ground without pattern. $P=10, r_{1}=3.42, r_{2}=2.92$, $r_{3}=4.6, r_{4}=4.1, w_{1}=w_{2}=0.5$ (Unit: $\left.\mathrm{mm}\right)$. ficient of cross-polarized waves and co-polarized waves, respectively. The azimuth angle $\psi$ and ellipticity angle $\kappa$ have been introduced to approve the polarization conversion. They are respectively expressed as follows:

$$
\begin{gathered}
\psi=\frac{1}{2} \arctan \left[\frac{2 R \cos (\zeta)}{1-R^{2}}\right], \\
\kappa=\frac{1}{2} \arcsin \left[\frac{2 R \sin (\zeta)}{1+R^{2}}\right], \\
R=\frac{\left|S_{\text {cross }-11}\right|}{\left|S_{\text {co-11 }}\right|}, \\
\zeta=\arg \left(S_{\text {cross }-11}\right)-\arg \left(S_{\text {co-11 }}\right) .
\end{gathered}
$$

Figure 2(a) shows the reflectance at normal incidence for the converter. It can be seen that the polarization converter performs ultra-broad bandwidth from $5.61 \mathrm{GHz}$ to $14.91 \mathrm{GHz}$ with $R_{\mathrm{xx}}, 0<-10 \mathrm{~dB}$ and $R_{\mathrm{xy}}, 0 \approx 0$. The phase results of $R_{\mathrm{xx}}$ and $R_{\mathrm{xy}}$ for the proposed converter are given in Fig. 2(b). Figure 2(c) shows the simulated PCRs as a function of frequency. It is shown that the PCRs of the proposed structure in Fig. 2(c) is roughly above 90\% from $5.71 \mathrm{GHz}$ to $15.02 \mathrm{GHz}$. From Fig. 2(d), $\psi$ is approximating to $90 \mathrm{deg}$ in the range of $5.09 \mathrm{GHz} 14.74 \mathrm{GHz}$ and $\kappa$ is larger than $-45 \mathrm{deg}$ and smaller than $45 \mathrm{deg}$ in the same range. Hence, it can be concluded that the polarization converter exhibits ultra-wide band cross polarization conversion from $5.71 \mathrm{GHz}$ to $14.91 \mathrm{GHz}$.

To illustrate the effect of the DMSRs, Figure 3 shows the surface current distributions of metallic structure at $6 \mathrm{GHz}$ and $14 \mathrm{GHz}$. The stronger surface current density is exhibited for DMSRs and the less surface current density is performed for copper ground plane. Also, it can be seen that the current density of the DMSR-1 at $6 \mathrm{GHz}$ and $14 \mathrm{GHz}$ are all more than that of DMSR-2 at $14 \mathrm{GHz}$. This phenomenon indicates that the response with different frequency can be obtained for TLW-PC due to the different parts. At the low frequency, the response is mainly caused by the DMSR-1 and DMSR-2. Correspondingly, TLW-PC achieves resonance at high frequency due to the DMSR-1. Therefore, DMSRs are all necessary for TLW-PC to obtain the ultra-wide band working frequency.

In order to demonstrate the manipulation for different polarized waves, the simulated magnetic fields of TLW-PC are given in Fig. 4 at $6 \mathrm{GHz}$ and $14 \mathrm{GHz}$. The magnetic resonance for DMSR-1 is exhibited at these frequencies. As shown in Fig. 4(a), the similar magnetic dipole resonances can be achieved for TE and TM polarized incidences. When the frequency is $14 \mathrm{GHz}$, the electronic dipole resonances are mainly caused by the DMSR-1 and the coupling effects between the DMSRs. It is clear that the TLW-PC controls the scattering waves both for TE polarized incidences and for TM polarized incident waves because the magnetic fields with different polarizations are same with each other. It is necessary to note that the manipulation with different polarizations maintained by the proposed TLW-PC is mainly attributed to the proposed DMSRs structure. 


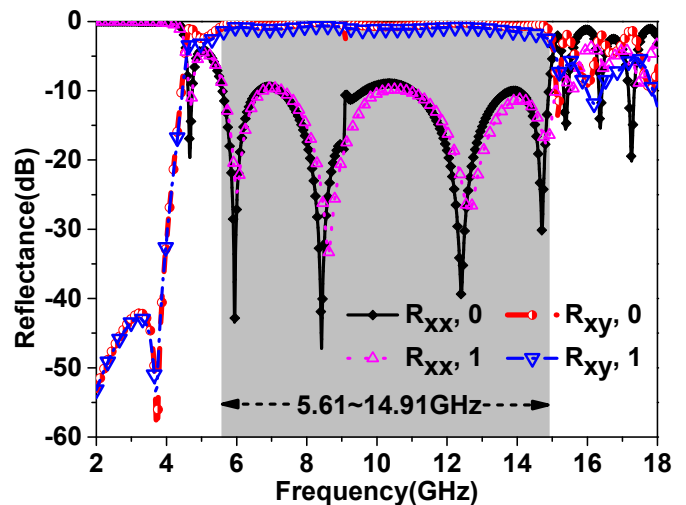

(a)

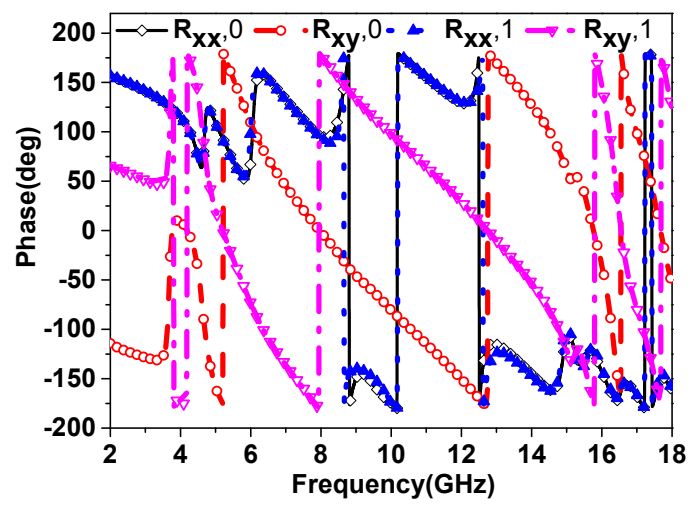

(b)

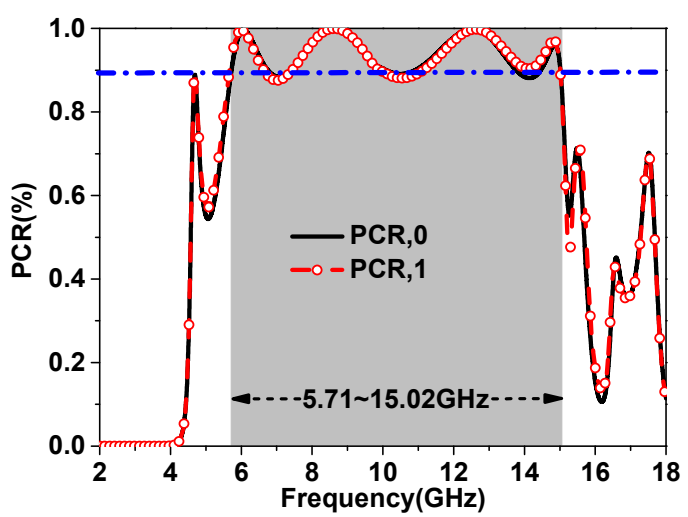

(c)

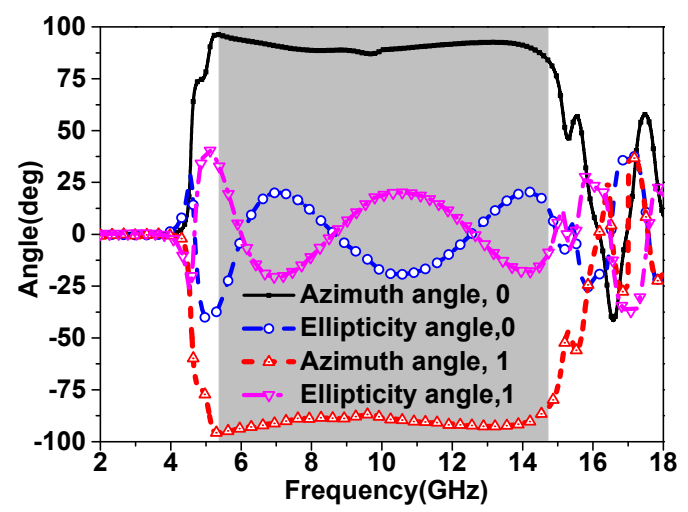

(d)

Fig. 2. Simulated results with cross-polarized and co-polarized waves for elements " 0 " and "1" in Fig. 1. (a) The amplitude of the reflectance. (b) The angle of the reflectance. (c) The PCR results. (d) The azimuth angle and ellipticity angle results.
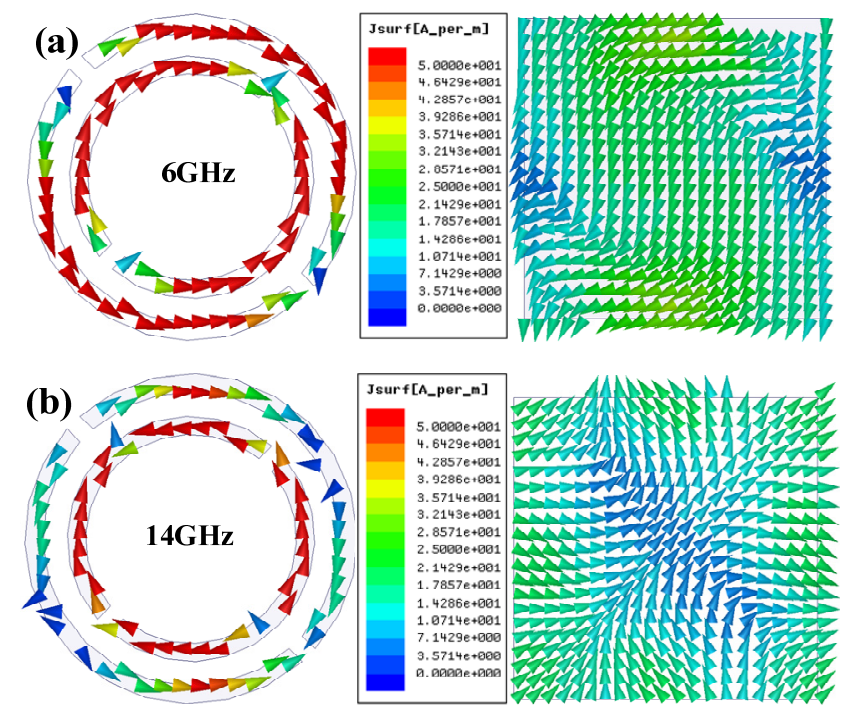

Fig. 3. Simulated surface current distributions of DMSRs and the copper ground plane at (a) $6 \mathrm{GHz}$ and (b) $14 \mathrm{GHz}$.
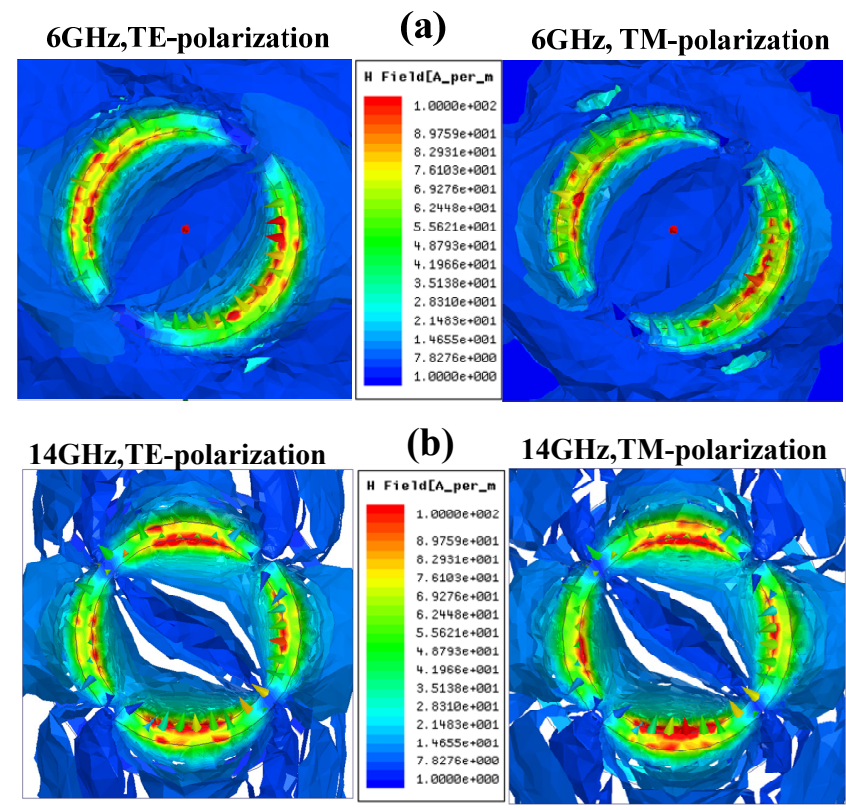

Fig. 4. Simulated magnetic fields of TLW-PC with different polarization at (a) $6 \mathrm{GHz}$ and (b) $14 \mathrm{GHz}$.

\section{Simulation and Analysis of Screen}

The metamaterial screen based on the TLW-PC is shown in Fig. 5. We consider a special metamaterial that is composed of binary digital elements of " 0 " and " 1 ". The element " 0 " is designed as the polarization converter shown in Fig. 1 and the element "1" is designed as the similar converter given in Fig. 5.

To satisfy the periodic boundary condition, $3 \times 3$ elements with the same dimension and structure are necessary to design the metamaterial screen. The physical realization of the digital elements is not unique, but it requires distinct responses to significant phase changes to have considerable freedom to control EM waves. The amplitude and phase of 


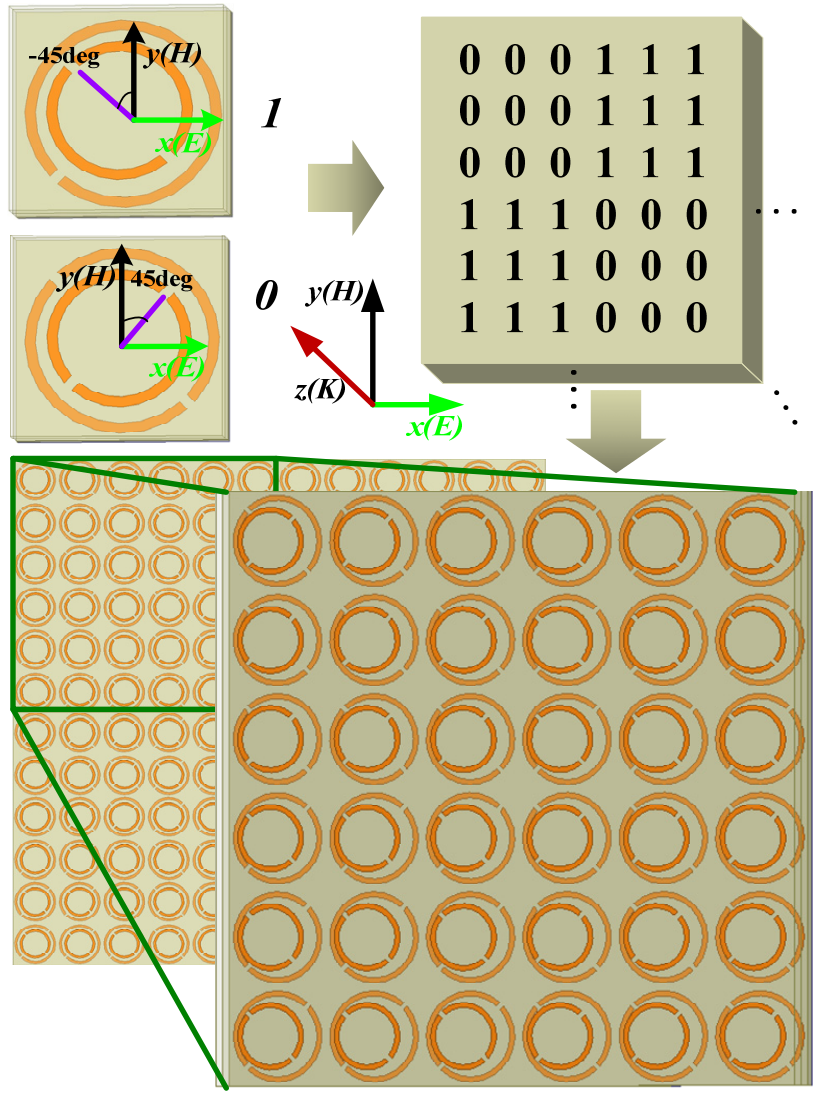

Fig. 5. The metamaterial screen based on polarization converters. The element " 0 " is designed as the polarization converter shown in Fig. 1 and the element " 1 " is designed as the polarization converter " 0 " with 90 deg rotation.

reflectance for element " 1 " are shown in Fig. 2. The phase difference between the elements " 0 " and " 1 " is given in Fig. 6. From Fig. 6, it can be seen that the TLW-PC as element "1" obtains ultra-wide bandwidth from $5.61 \mathrm{GHz}$ to $14.91 \mathrm{GHz}$ with $R_{\mathrm{xx}}, 1<-10 \mathrm{~dB}$ and $R_{\mathrm{xy}}, 1 \approx 0$. It means that the polarization of reflected EM waves can be rotated by the element " 1 ". The phase difference $\Delta \varphi$ within elements " 0 " and " 1 " is defined as

$$
\Delta \varphi=\left|\varphi_{R_{x y}, 0}-\varphi_{R_{x y}, 1}\right|
$$

where $\varphi_{R_{x y}, 0}$ and $\varphi_{R_{x y}, 1}$ are the phase of cross-polarized waves for elements of " 0 " and " 1 ". From Fig. 4 , it is observed that $\Delta \varphi$ is shifted between $172 \mathrm{deg}$ and $184 \mathrm{deg}$ from $2 \mathrm{GHz}$ to $18 \mathrm{GHz}$. Importantly, the elements alter the phases, amplitudes of reflected waves. The destructive interference is exhibited due to the phase difference. The wideband low scattering can be obtained which attributes to destructive interference of EM wave with superposition. Hence, the scattering characteristics would be improved for the proposed metamaterial screen.

The low scattering characteristics of monostatic RCS reduction for the metamaterial screen are shown in Fig. 7(a) compared with that of the proposed polarization converter " 0 ". We can see that the monostatic RCS reduction can be obtained from 4.65 to $17.82 \mathrm{GHz}$ with $5 \mathrm{~dB}$ and

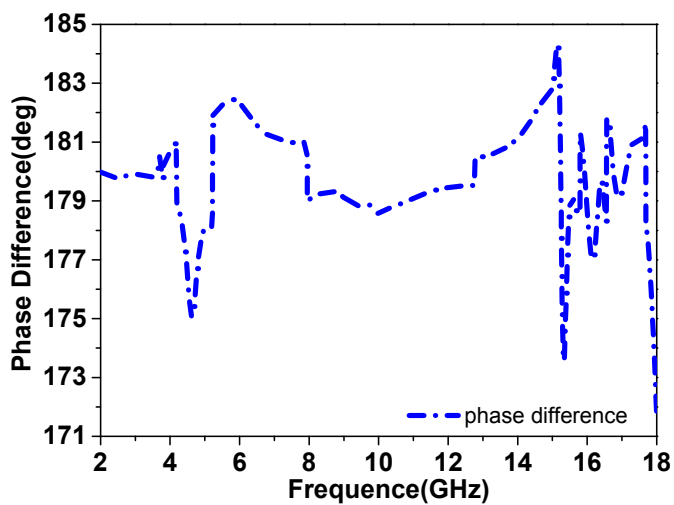

Fig. 6. The simulated results of the phase difference between elements of " 0 " and " 1 ".

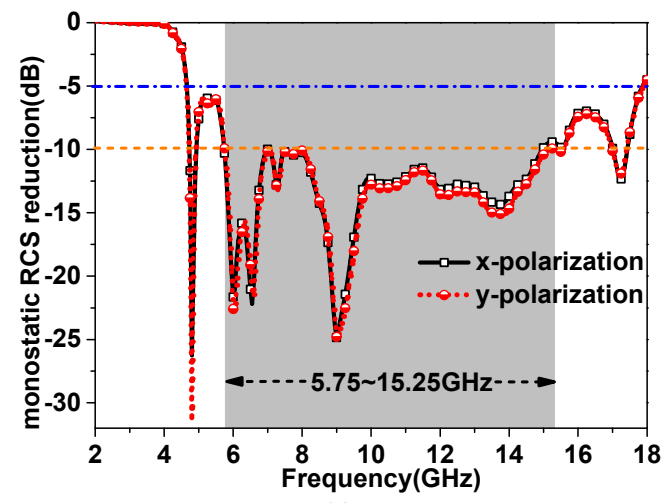

(a)
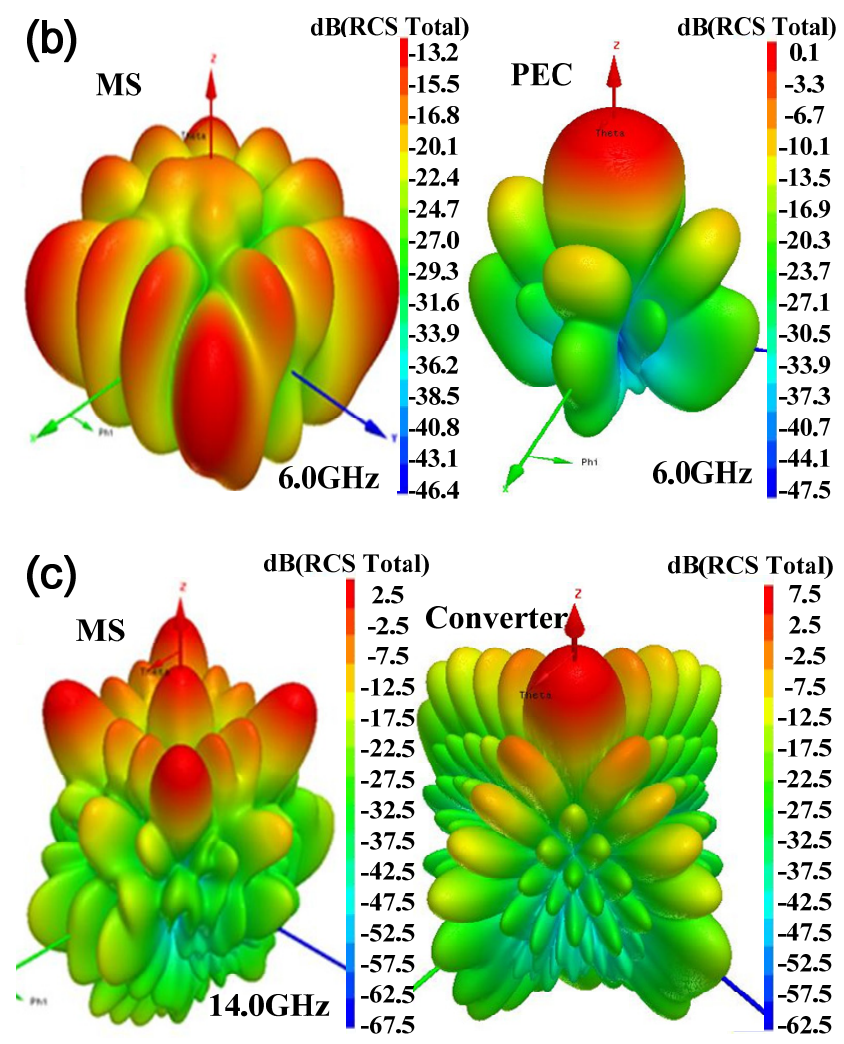

Fig. 7. (a) Monostatic RCS reduction of the metamaterial screen (MS) compared with the converter in Fig. 1. The bistatic RCS patterns of HMS and the converter at (b) $6 \mathrm{GHz}$ and (c) $14 \mathrm{GHz}$. 

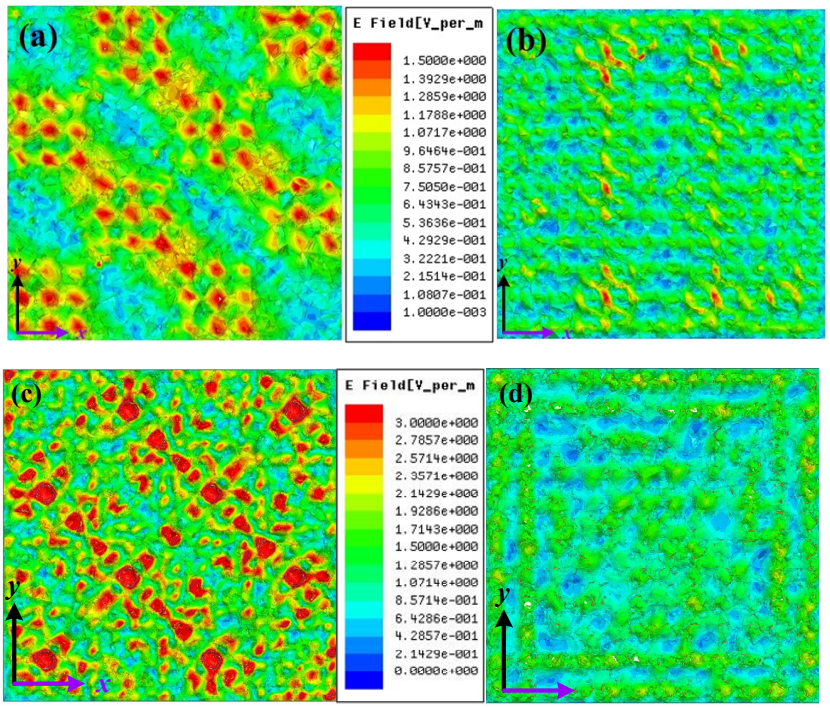

Fig. 8. Near scattering electronic fields at $6.0 \mathrm{GHz}$ for (a) MS and (b) the converter " 0 " in Fig. 1, and $14 \mathrm{GHz}$ for (c) MS and (d) the converter "0" in Fig. 1.

from 5.75 to $15.25 \mathrm{GHz}$ with $10 \mathrm{~dB}$ for the metamaterial screen. To illustrate this physical phenomenon, the three dimensional bistatic RCS patterns for the screen and the converter are given in Figs. 7(b) and 7(c) at $6 \mathrm{GHz}$ and $14 \mathrm{GHz}$. The scattering beams of the screen have been visibly changed compared with that of converter due to the phase difference. For converter, the polarizations of scattering waves have been varied, whereas the total scattering fields are not decreased. The scattering beam direction of converter is along the $\mathrm{z}$ axis, which is similar to the perfect conductor. For the metamaterial screen, the scattering fields have been reduced by the phase difference. The beam of the screen is synthesized by the elements of " 0 " and " 1 " and the phase differences significantly alter the beam direction.

For illustrating the mechanism of controlling the scattering fields by the metamaterial screen, the comparison of near scattering electric fields for the metamaterial screen and the converter " 0 " were simulated and shown in Fig. 8 at $6.0 \mathrm{GHz}$ and $14.0 \mathrm{GHz}$. It can be observed from Fig. 8(a) and 8(c) that the stronger resonant electric fields are obtained for element of " 1 " and the weak ones are achieved by the element of " 0 " at $6 \mathrm{GHz}$ and $14 \mathrm{GHz}$. The remarkable differences of near scattering field are attributed to the phase difference between elements of " 0 " and " 1 " and the distinct structure. The scattering electric far-fields have been manipulated due to the destructive interference which is attributed to the control of phase and amplitude by the TLW-PC. In a word, it is phase difference that makes the variety of scattering beams, the change of near scattering fields and the low scattering characteristics for the metamaterial screen.

\section{Fabrication and Measurement}

To validate the performances mentioned above, a metamaterial screen device with $576(24 \times 24)$ cells and

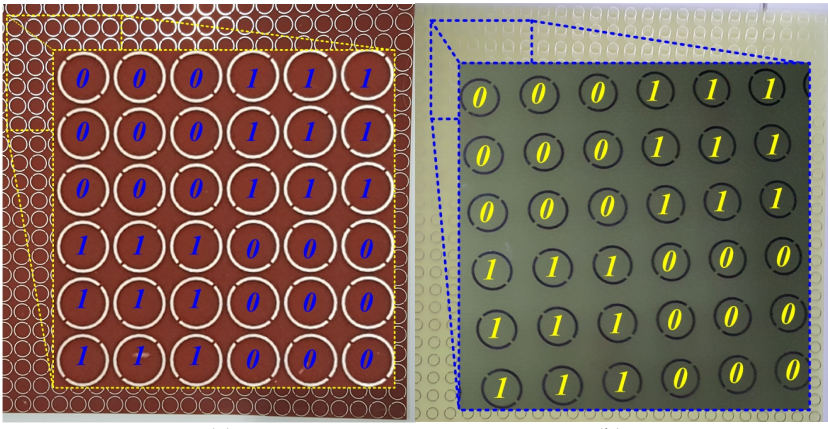

(a)

(b)

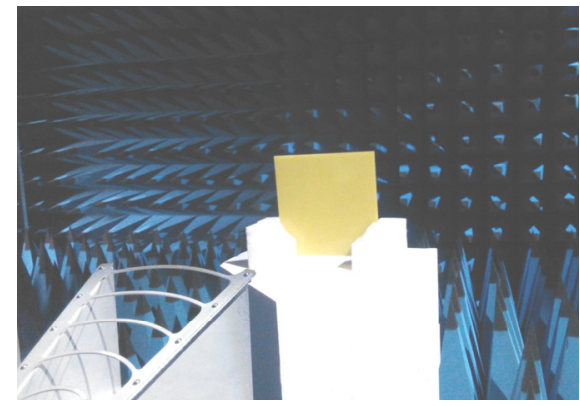

(c)

Fig. 9. The metamaterial screen device and measurement in a microwave anechoic chamber. (a) Bottom layer of the metamaterial screen device. (b) Second layer of the device. (c) The metamaterial screen device measured in a microwave anechoic chamber.

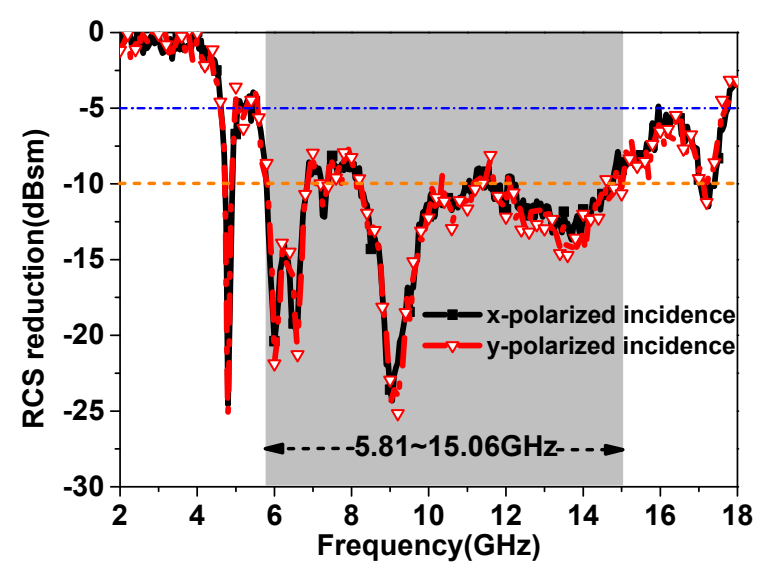

Fig. 10. Experimental results of RCS reduction for the metamaterial screen device with different polarized incidences.

the converter device with the same area have been fabricated using the common printed circuit board processing technology. They have been tested by employing the freespace test method in a microwave anechoic chamber. The proposed metamaterial screen device is shown in Fig. 9. The dielectric substrate was chosen as FR4 boards with the thicknesses of $1.5 \mathrm{~mm}, 1.5 \mathrm{~mm}$ and $1 \mathrm{~mm}$. The metal DMSRs and ground were made of $0.035 \mathrm{~mm}$-thick copper layers. A vector network analyzer (Agilent N5230C) and two standard-gain horn antennas were used to transmit and receive the EM waves. Two devices with same area were placed vertically in the center of a turntable to ensure that the incidences were similar to a plane wave for measuring scattering characteristics. 
The experimental results of RCS reduction are given in Fig. 10. More in details, the metamaterial device exhibits an ultra-broadband low scattering from 5.81 to $15.06 \mathrm{GHz}$ of $10 \mathrm{~dB}$ RCS reduction and from 4.72 to $17.65 \mathrm{GHz}$ of $5 \mathrm{~dB}$ RCS reduction for different polarized waves. Moreover, several RCS reduction peaks can be obtained from Fig. 10. The similar RCS reduction cures for different polarized incidences indicate the insensitive polarization for the metamaterial screen device. Experimental results agree well with simulated results. It is noted that the differences between the simulation and the measurement are addressed by the gaps in different layers.

\section{Conclusion}

In conclusion, we proposed a metamaterial screen device composed of a three layers broadband polarization converter. The mechanism of incident electromagnetic wave control was theoretically illustrated and the characteristics of low scattering for the device were demonstrated numerically and experimentally. The results indicated that the device exhibited ultra-broadband RCS reduction of $10 \mathrm{~dB}$ from $5.81 \mathrm{GHz}$ to $15.06 \mathrm{GHz}$. Good agreements between the simulation and measurement were observed. The metamaterial device can be applicable to the stealthy technology.

\section{Acknowledgments}

This work is partially supported by the National Natural Science Foundation of China under grant (No. 61501494, 61471389, 61271100), the Natural Science Foundational Research Fund of Shaanxi Province (No. 2012JM8003), and the Doctoral Foundation of Air Force Engineering University under grant (No. KGD080914002). The authors also thank the reviewers for their valuable comments.

\section{References}

[1] ALÙ, A. Mantle cloak: Invisibility induced by a surface. Physical Review B, 2009, vol. 80, p. 245115-1-245115-5. DOI: 10.1103/PhysRevB.80.245115

[2] MONTI, A., SORIC, J., BARBUTO, M., et al. Mantle cloaking for co-site radio-frequency antennas. Applied Physics Letters, 2016, vol. 108 , no. 11 , p. 113502-1-113502-5. DOI: 10.1063/1.4944042

[3] ALITALO, P., LUUKKONEN, O., JYLHA, L., et al. Transmission-line networks cloaking objects from electromagnetic fields. IEEE Transactions on Antennas and Propagation, 2008, vol. 56, no. 2, p. 416-424. DOI: 10.1109/TAP. 2007.915469

[4] PENDRY, J. B., SCHURIG, D., SMITH, D. R. Controlling electromagnetic fields. Science, 2006, vol. 312, no. 5781, p. 1780 to 1782 . DOI. $10.1126 /$ science. 1125907

[5] SMITH, D. R., PENDRY, J. B., WILTSHIRE, M. C. K. Metamaterials and negative refractive index. Science, 2004, vol. 305 , no. 5685 , p. $788-792$. DOI: $10.1126 /$ science. 1096796

[6] WAN, X., LI, Y. B., CAI, B. G., et al. Simultaneous controls of surface waves and propagating waves by metasurfaces. Applied Physics Letters, 2014, vol. 105, no. 12, p. 121603-1-121603-5. DOI: $10.1063 / 1.4896540$

[7] NI, X. J., WONG, Z. J., MREJEN, M., et al. An ultrathin invisibility shin cloak for visible light. Science, 2015, vol. 349, no. 6254 , p. 1310-1314. DOI: $10.1126 /$ science.aac9411

[8] LI, S. J., GAO, J., CAO, X. Y., et.al. Multiband and broadband polarization-insensitive perfect absorber devices based on a tunable and thin double split-ring metamaterial. Optics Express, 2015, vol. 23 , no. 3, p. 3523-3533. DOI: 10.1364/OE.23. 003523

[9] VASA, P., WANG, W., POMRAENKE, R., et al. Optical Stark effects in J-aggregate-metal hybrid nanostructures exhibiting a strong exciton-surface-plasmon-polariton interaction. Physical Review Letters, 2015, vol. 114, no. 3, p. 036802-1-036802-6. DOI: 10.1103/PhysRevLett.114.036802

[10] LANDY, N. I., SAJUYIGBE, S., MOCK, J. J., et al. A perfect metamaterial absorber. Physical Review Letters, 2008, vol. 100, p. 207402-1-207402-4. DOI: 10.1103/PhysRevLett.100.207402

[11] LI, S., GAO, J., CAO, X., et al. Wideband, thin, and polarizationinsensitive perfect absorber based the double octagonal rings metamaterials and lumped resistances. Journal of Applied Physics, 2014, vol. 116 , no. 4, p. 043710-1-043710-6. DOI: $10.1063 / 1.4891716$

[12] LI, S. J., GAO, J., CAO, X. Y., et al. Loaded metamaterial perfect absorber using substrate integrated cavity. Journal of Applied Physics, 2014, vol. 115, no. 21, p. 213703-1-213703-5. DOI: $10.1063 / 1.4881115$

[13] SUN, L. K., CHENG, H. F., ZHOU, Y. J., et al. Broadband metamaterial absorber based on coupling resistive frequency selective surface. Optics Express, 2012, vol. 20, no. 4, p. 4675-4680. DOI: 10.1364/OE.20.004675

[14] YOO, M., LIM, S. Polarization-independent and ultra-wide band metamaterial absorber using a hexagonal artificial impedance surface and a resistor-capacitor layer. IEEE Transactions on Antennas and Propagation, 2014, vol. 62, no. 5, p. 2652-2658. DOI: 10.1109/TAP.2014.2308511

[15] LI, S. J., GAO, J., CAO, X. Y., et al. Polarization-insensitive and thin stereometamaterial with broadband angular absorption for oblique incidence. Applied Physics A, 2015, vol. 119, no. 1, p. 371-378. DOI: $10.1007 / \mathrm{s} 00339-014-8978-\mathrm{y}$

[16] PAQUAY, M., IRIARTE, J. C., EDERRA, I., et al. Thin AMC structure for radar cross-section reduction. IEEE Transactions on Antennas and Propagation, 2007, vol. 55, no. 12, p. 3630-3638. DOI: 10.1109/TAP.2007.910306

[17] GENOVESI, S., COSTA, F., MONORCHIO, A. Wideband radar cross section reduction of slot antennas arrays. IEEE Transactions on Antennas and Propagation, 2014, vol. 62, no. 1, p. 163-173. DOI: 10.1109/TAP.2013.2287888

[18] IRIARTE GALARREGUI, J. C., TELlECHEA PEREDA, A., MARTINEZ DE FALCÓN, J. L., et al. Broadband radar crosssection reduction using AMC technology. IEEE Transactions on Antennas and Propagation, 2013, vol. 61, no. 12, p. 6136-6143. DOI: 10.1109/TAP.2013.2282915

[19] HAN, J. F., CAO, X. G., GAO, J., et al. Design of broadband reflective 90deg polarization rotator based on metamaterial. Acta Physica Sinica, 2016, vol. 65, no. 4, p. 044201. DOI: 10.7498/aps.65.044201

[20] HUANG, X. J., XIAO, B. X., YANG, D., et al. Ultra-broadband 90deg polarization rotator based on bi-anisotropic metamaterial. Optics Communications, 2015, vol. 338, p. 416-421. DOI: 10.1016/j.optcom.2014.11.010 
[21] LI, S. J., GAO, J., CAO, X. Y., et al. Hybrid metamaterial device with wideband absorption and multiband transmission based on spoof surface plasmon polaritons and perfect absorber. Applied Physics Letters. 2015, vol. 106, no. 18, p. 181103. DOI: $10.1063 / 1.4919789$

[22] GAO, X., HAN, X., CAO, W. P., et al. Ultra-wideband and highefficiency linear polarization converter based on double $\mathrm{V}$-shaped metasurface. IEEE Transaction on Antennas and Propagation, 2015, vol. 63, no. 8, p. 3522-3530. DOI: 10.1109/TAP.2015.2434392

[23] YIN, J. Y., WAN, X., ZHANG, Q., et al. Ultra wideband polarization-selective conversions of electromagnetic waves by metasurface under large-range incident angles. Scientific Reports, 2015, vol. 5, 10 p. DOI: $10.1038 /$ srep 12476

[24] JIA, Y., LIU, Y., GUO, Y. J., et al. Broadband polarization rotation reflective surfaces and their applications to RCS reduction. IEEE Transaction on Antennas and Propagation, 2016, vol. 64, no. 1, p. 179-185. DOI: 10.1109/TAP.2015.2502981

[25] PAN, W. B., HUANG, C., PU, M. B., et al. Combining the absorptive and radiative loss in metasurface for multi-spectral shaping of the electromagnetic scattering. Scientific Reports, 2016, vol. 6,8 p. DOI: $10.1038 /$ srep2 1462

[26] ZHAO, Y., CAO, X. G., GAO, J., et al. Broadband diffusion metasurface based on a single anisotropic element and optimized by the Simulated Annealing algorithm. Scientific Reports, 2016, vol. 6, 9 p. DOI:10.1038/srep23896

[27] LI, S., CAO, X., XU, L., et al. Ultra-broadband reflective metamaterial with RCS reduction based on polarization convertor, information entropy theory and genetic optimization algorithm. Scientific Reports, 2016, vol. 6, 12 p. DOI: 10.1038/srep37409

\section{About the Authors ...}

Si-Jia LI was born in Xi'an, Shaanxi province, P.R. China in 1987. He received the B. Eng. degree in Electronics and Information Engineering from Guangxi University, Nanning, China, in 2009, and the M. Eng. degree in Information and Telecommunication Engineering from the Air Force Engineering University, Xi'an China, in 2011. He received the Ph.D degree in Electronic Science and Technology at the Information and Navigation College, Air Force Engineering University in 2015. Now he is a senior researcher in the Air Force Engineering University.

He has been working with the Military Communication and Navigation Antenna and EMC Lab since 2012. His research activity has been focused in the metamaterial, the broadband and fractal perfect metamaterial absorber and its application for RCS reduction of antennas. He has authored and coauthored more than 60 scientific papers in major journals and international conferences. He received the excellent Master's dissertation in 2012. He is awarded the 2012, 2013 and 2014 Best Student Paper Prize (First Prize) of the National Graduate Mathematical Modeling Contest in Shanghai in December 2012, in Changsha in December 2013 and in Tianjin in December 2014. He was a recipient of the Best Paper Award at the Forth National Doctor Forum Symposium on Information Science of China in Guangzhou in October 2013. He is awarded the excellent student in the 2014 national summer school of the Microwave Material and Components. He is a reviewer of the Applied Physics Letter, Journal of Applied Physics, IEEE Transactions on Microwave Theory \& Techniques, IEEE Transactions on Antennas and Propagation, IEEE Antennas Wireless Propagation Letter, Electronic Letters and Microelectronics Journal.

Xiangyu CAO received the B. Sc and M.A. Sc degrees from the Air Force Missile Institute in 1986 and 1989, respectively. She joined the Air Force Missile Institute in 1989 as an assistant teacher. She became an associate professor in 1996. She received Ph.D. degree in the Missile Institute of Air Force Engineering University in 1999. From 1999 to 2002, she was engaged in postdoctoral research in Xidian University, China. She was a Senior Research Associate in the Dept. of Electronic Engineering, City University of Hong Kong from June 2002 to Dec 2003. She is currently a professor of Information and Navigation college, Air Force Engineering University of CPLA. She is the IEEE senior member from 2008.

She has authored and coauthored more than 200 technical journal articles and conference papers, and holds one China soft patent. She is the coauthor of two books entitled, Electromagnetic Field and Electromagnetic Wave, and Microwave Technology and Antenna published in 2007 and 2008, respectively. Her research interests include smart antennas, electromagnetic metamaterial and their antenna applications, and electromagnetic compatibility. She is a reviewer of the Applied Physics Letter, Journal of Applied Physics, IEEE Transactions on Antennas and Propagation, and IEEE Antennas Wireless Propagation Letter. 